\title{
Cyclic permutations of sequences and uniform partitions
}

\author{
Po-Yi Huang* \\ Department of Mathematics \\ National Cheng Kung University \\ Tainan, Taiwan \\ pyhuang@mail.ncku.edu.tw
}

\author{
Jun $\mathrm{Ma}^{\dagger}$ \\ Department of Mathematics \\ Shanghai Jiao Tong University \\ Shanghai, China \\ majun904@sjtu.edu.cn
}

\author{
Yeong-Nan Yeh \\ Institute of Mathematics \\ Academia Sinica \\ Taipei, Taiwan \\ mayeh@math.sinica.edu.tw
}

Submitted: Apr 25, 2010; Accepted: Jul 28, 2010; Published: Aug 24, 2010

Mathematics Subject Classification: 05A18

\begin{abstract}
Let $\vec{r}=\left(r_{i}\right)_{i=1}^{n}$ be a sequence of real numbers of length $n$ with sum $s$. Let $s_{0}=0$ and $s_{i}=r_{1}+\ldots+r_{i}$ for every $i \in\{1,2, \ldots, n\}$. Fluctuation theory is the name given to that part of probability theory which deals with the fluctuations of the partial sums $s_{i}$. Define $p(\vec{r})$ to be the number of positive sum $s_{i}$ among $s_{1}, \ldots, s_{n}$ and $m(\vec{r})$ to be the smallest index $i$ with $s_{i}=\max _{0 \leqslant k \leqslant n} s_{k}$. An important problem in fluctuation theory is that of showing that in a random path the number of steps on the positive half-line has the same distribution as the index where the maximum is attained for the first time. In this paper, let $\vec{r}_{i}=\left(r_{i}, \ldots, r_{n}, r_{1}, \ldots, r_{i-1}\right)$ be the $i$-th cyclic permutation of $\vec{r}$. For $s>0$, we give the necessary and sufficient conditions for $\left\{m\left(\vec{r}_{i}\right) \mid 1 \leqslant i \leqslant n\right\}=\{1,2, \ldots, n\}$ and $\left\{p\left(\vec{r}_{i}\right) \mid 1 \leqslant i \leqslant n\right\}=\{1,2, \ldots, n\}$; for $s \leqslant 0$, we give the necessary and sufficient conditions for $\left\{m\left(\vec{r}_{i}\right) \mid 1 \leqslant i \leqslant\right.$ $n\}=\{0,1, \ldots, n-1\}$ and $\left\{p\left(\vec{r}_{i}\right) \mid 1 \leqslant i \leqslant n\right\}=\{0,1, \ldots, n-1\}$. We also give an analogous result for the class of all permutations of $\vec{r}$.
\end{abstract}

Keywords: Cyclic permutation; Fluctuation theory; Uniform partition

\footnotetext{
*Partially supported by NSC 96-2115-M-006-012

${ }^{\dagger}$ Corresponding author

${ }^{\ddagger}$ Partially supported by NSC 96-2115-M-001-005
} 


\section{Introduction}

Fluctuation theory is the name given to that part of probability theory which deals with the fluctuations of the partial sums $s_{i}=x_{1}+\ldots+x_{i}$ of a sequence of random variables $x_{1}, \ldots, x_{n}$. An important problem in fluctuation theory is that of showing that in a random path the number of steps on the positive half-line has the same distribution as the index where the maximum is attained for the first time. In particular, fix a sequence of real numbers $\vec{r}=\left(r_{i}\right)_{i=1}^{n}=\left(r_{1}, \ldots, r_{n}\right)$. Let

$$
s_{0}=0, s_{1}=r_{1}, s_{2}=r_{1}+r_{2}, \ldots, s_{n}=r_{1}+r_{2}+\ldots+r_{n}
$$

Define $p(\vec{r})$ to be the number of positive sums $s_{i}$ among $s_{1}, \ldots, s_{n}$, i.e., $p(\vec{r})=\left|\left\{i \mid s_{i}>0\right\}\right|$, and $m(\vec{r})$ to be the smallest index $i$ with $s_{i}=\max _{0 \leqslant k \leqslant n} s_{k}$. Let $[n]$ and $[n]-1$ denote the sets $\{1,2, \ldots, n\}$ and $\{0,1, \ldots, n-1\}$, respectively. Let $\mathfrak{S}_{n}$ be the set of all the permutations on the set $[n]$. We write permutations of $\mathfrak{S}_{n}$ in the form $\sigma=(\sigma(1) \sigma(2) \cdots \sigma(n))$. Let $\vec{r}_{\sigma}=\left(r_{\sigma(1)}, \ldots, r_{\sigma(n)}\right)$ for any $\sigma \in \mathfrak{S}_{n}$. For any $i \in[n+1]-1$, Let $N(\vec{r} ; i)(\operatorname{resp} . \Pi(\vec{r} ; i))$ be the number of permutations $\sigma$ in $\mathfrak{S}_{n}$ such that $p\left(\vec{r}_{\sigma}\right)=i$ (resp. $m\left(\vec{r}_{\sigma}\right)=i$ ). A basic theorem in fluctuation theory states that $N(\vec{r} ; i)=\Pi(\vec{r} ; i)$ for any $i \in[n+1]-1$. This result first was proved by Andersen [2]. Feller [10] called this result the Equivalence Principle and gave a simpler proof. This result is mentioned by Spitzer [23]. Baxter [3] obtained this result by bijection method. In [4], Brandt generalized the Equivalence Principle. Hobby and Pyke in [12] and Altschul in [1] gave bijection proofs for the generalization of Brandt.

Given an index $i \in[n]$, let $\vec{r}_{i}=\left(r_{i}, \ldots, r_{n}, r_{1}, \ldots, r_{i-1}\right)$. We call $\vec{r}_{i}$ the $i$-th cyclic permutation of $\vec{r}$. Let

$$
\mathcal{P}(\vec{r})=\left\{p\left(\vec{r}_{i}\right) \mid i \in[n]\right\} \quad \text { and } \quad \mathcal{M}(\vec{r})=\left\{m\left(\vec{r}_{i}\right) \mid i \in[n]\right\} .
$$

Spitzer [23] showed implicitly the following specialization of the Equivalence Principle to the case of cyclic permutations.

Lemma 1.1 (Spitzer combinatorial lemma, [23]) Let $\vec{r}$ be a sequence of real numbers of length $n$ with sum 0 and the partial sums $s_{1}, \ldots, s_{n}$ are all distinct. Then $\mathcal{P}(\vec{r})=\mathcal{M}(\vec{r})=$ $[n]-1$.

A set is uniformly partitioned if all partition classes have the same cardinality. Many uniform partitions of combinatorial structures are consequences of Lemma 1.1. A famous example is the Chung-Feller theorem. Let $\mathscr{D}$ be the set of sequences of integers $\vec{r}=\left(r_{i}\right)_{i=1}^{2 n}$ such that $s_{2 n}=0$ and $r_{i} \in\{1,-1\}$ for all $i \in[2 n]$. Clearly, $|\mathscr{D}|=\left(\begin{array}{c}2 n \\ n\end{array}\right)$. The Chung-Feller theorem shows that $n+1$ divides $\left(\begin{array}{c}2 n \\ n\end{array}\right)$ by uniformly partitioning the set $\mathscr{D}$ into $n+1$ classes.

The Chung-Feller theorem was proved by many different methods. Chung and Feller [7] obtained this result by analytic methods. Narayana [19] showed this theorem by combinatorial methods. Narayana's book [20] introduced a refinement of this theorem. 
Mohanty's book [18] devotes an entire section to exploring this theorem. Callan in [5] and Jewett and Ross in [14] gave bijection proofs of this theorem. Callan [6] reviewed and compared combinatorial interpretations of three different expressions for the Catalan number by cycle method.

One also attempted to generalize the Chung-Feller theorem for finding uniformly partitions of other combinatorial structures. Huq [13] developed generalized versions of this theorem for lattice paths. Eu, Liu and Yeh [9] proved this Theorem by using the Taylor expansions of generating functions and gave a refinement of this theorem. In [8], Eu, Fu and Yeh gave a strengthening of this Theorem and a weighted version for Schröder paths.

Suppose $f(x)$ is a generating function for some combinatorial sequences. Let $F(x, y)=$ $\frac{y f(x y)-f(x)}{y-1}$. Liu, Wang and Yeh [15] call $F(x, y)$ the function of Chung-Feller type for $f(x)$. If we can give a combinatorial interpretation for the function $F(x, y)$, then we may uniformly partition the set formed by this combinatorial structure. Ma and Yeh [16] attempted to find combinatorial interpretation of the function of Chung-Feller type for a generating function of three classes of different lattice paths.

Particularly, Narayana [19] showed the following property for cyclic permutations.

Lemma 1.2 (Narayana [19]) Let $\vec{r}=\left(r_{i}\right)_{i=1}^{n}$ be a sequence of integers with sum 1 . Then $\mathcal{P}(\vec{r})=[n]$.

In [19], Narayana gave a combinatorial proof of the Chung-Feller theorem by Lemma 1.2 and uniformly partition the set $\mathscr{D}$. Lemma 1.2 is derivable as a special case from the Spitzer combinatorial lemma. In [17], Ma and Yeh gave a generalizations of Lemma 1.2 by considering $\lambda$-cyclic permutations of a sequence of vectors and uniformly partition sets of many new combinatorial structures.

Based on the rightmost lowest point of a lattice path, Woan [24] presented another new uniform partition of the set $\mathscr{D}$. Let $\mathscr{B}$ be the set of sequences of integers $\vec{r}=\left(r_{i}\right)_{i=1}^{n+1}$ such that $s_{n+1}=1$ and $r_{i} \in\{1,0,-1\}$ for all $i \in[n+1]$. In [9], Eu, Liu and Yeh proved that there is an uniform partition for the set $\mathscr{B}$, which was found by Shapiro [22]. In [17], Ma and Yeh also proved another interesting property of cyclic permutations as follows.

Lemma 1.3 Let $\vec{r}=\left(r_{i}\right)_{i=1}^{n}$ be a sequence of integers with sum 1 . Then $\mathcal{M}(\vec{r})=[n]$.

Raney [21] discovered a fact: If $\vec{r}=\left(r_{i}\right)_{i=1}^{n}$ is any sequence of integers whose sum is 1 , then exactly one of the cyclic permutations has all of its partial sums positive. Graham and Knuth's book [11] introduced a simple geometric argument of the results obtained by Raney. This geometric argument yields $\mathcal{P}(\vec{r})=\mathcal{M}(\vec{r})=[n]$ for integer sequences $\vec{r}$ with sum 1.

Fix a sequence of real numbers $\vec{r}=\left(r_{i}\right)_{i=1}^{n}$ with sum $s$. For $s=0$, Lemma 1.1 give a characterization for $\mathcal{P}(\vec{r})=[n]-1$; we note that the conditions in Lemma 1.1 are not necessary for $\mathcal{M}(\vec{r})=[n]-1$. For example, let $\vec{r}=(0,1,-1)$. We have $\mathcal{M}(\vec{r})=\{0,1,2\}$ and $\mathcal{P}(\vec{r})=\{0,1\}$. For $s=1$, Lemmas 1.2 and 1.3 give some sufficient conditions for $\mathcal{P}(\vec{r})=[n]$ and $\mathcal{M}(\vec{r})=[n]$ respectively. Note that $\mathcal{M}(\vec{r}) \subseteq[n]$ and $\mathcal{P}(\vec{r}) \subseteq[n]$ if $s>0$; $\mathcal{M}(\vec{r}) \subseteq[n]-1$ and $\mathcal{P}(\vec{r}) \subseteq[n]-1$ if $s \leqslant 0$. Two natural problems arise: 
(1) What are necessary and sufficient conditions for $\mathcal{M}(\vec{r})=[n]$ and $\mathcal{P}(\vec{r})=[n]$ if $s>0$ ?

(2) What are necessary and sufficient conditions for $\mathcal{M}(\vec{r})=[n]-1$ and $\mathcal{P}(\vec{r})=[n]-1$ if $s \leqslant 0$ ?

The aim of this paper is to solve these two problems. Let $\vec{r}=\left(r_{i}\right)_{i=1}^{n}$ be a sequence of real numbers with sum $s$ and partial sums $\left(s_{i}\right)_{i=0}^{n}$. We state the main results of this paper as follows.

- Let $s>0$. Then

(1) $\mathcal{M}(\vec{r})=[n]$ if and only if $s_{j}-s_{i} \geqslant s$ for all $1 \leqslant i \leqslant j-1$ with $j=m(\vec{r})$.

(2) $\mathcal{P}(\vec{r})=[n]$ if and only if $s_{j}-s_{i} \notin(0, s)$ for any $1 \leqslant i<j \leqslant n$, where the notation $(0, s)$ denote the set of all real numbers $x$ satisfying $0<x<s$.

- Let $s \leqslant 0$. Then

(1) $\mathcal{M}(\vec{r})=[n]-1$ if and only if $s_{i}-s_{j}<s$ for all $j+1 \leqslant i \leqslant n-1$ with $j=m(\vec{r})$.

(2) $\mathcal{P}(\vec{r})=[n]-1$ if and only if $s_{j}-s_{i} \notin[s, 0]$ for all $1 \leqslant i<j \leqslant n$, where the notation $[s, 0]$ denote the set of all real numbers $x$ satisfying $s \leqslant x \leqslant 0$.

The properties of cyclic permutations of the sequence $\vec{r}$ in the main results will be proved in Section 2. Lemmas 1.1, 1.2 and 1.3 are corollaries of the main results.

Recall that $N(\vec{r} ; i)$ ( resp. $\Pi(\vec{r} ; i)$ ) denotes the number of permutations $\sigma$ in $\mathfrak{S}_{n}$ such that $p\left(\vec{r}_{\sigma}\right)=i$ (resp. $m\left(\vec{r}_{\sigma}\right)=i$ ). Using the main results, we derive the necessary and sufficient conditions of $N(\vec{r} ; i)=\Pi(\vec{r} ; i)=(n-1)$ ! for all $i \in[n]$ (resp. $i \in[n]-1)$ when $s>0$ (resp. $s \leqslant 0)$.

We also consider more general cases. Fix a real number $\theta$. Define $p(\vec{r} ; \theta)$ to be the number of sum $s_{i}$ among $s_{1}, \ldots, s_{n}$ such that $s_{i}>\theta \cdot i$. Let $\mathcal{P}(\vec{r} ; \theta)=\left\{p\left(\vec{r}_{i} ; \theta\right) \mid i \in[n]\right\}$. Define $m(\vec{r} ; \theta)$ to be the smallest index $i$ with $s_{i}-\theta \cdot i=\max _{0 \leqslant k \leqslant n}\left(s_{k}-\theta \cdot k\right)$. Let $\mathcal{M}(\vec{r} ; \theta)=$ $\left\{m\left(\vec{r}_{i} ; \theta\right) \mid i \in[n]\right\}$. Suppose $s>n \theta$. We give the necessary and sufficient conditions for $\mathcal{M}(\vec{r} ; \theta)=[n]$ and $\mathcal{P}(\vec{r} ; \theta)=[n]$. Suppose $s \leqslant n \theta$. We give the necessary and sufficient conditions for $\mathcal{M}(\vec{r} ; \theta)=[n]-1$ and $\mathcal{P}(\vec{r} ; \theta)=[n]-1$.

We organize this paper as follows. In Section 2, we study properties of cyclic permutations of $\vec{r}$. In Section 3, we consider more general cases.

\section{Properties of cyclic permutations of a sequence}

In this section, we study properties of cyclic permutations of a sequence $\vec{r}$ with sum $s$. For $s>0$, we give the necessary and sufficient conditions for $\mathcal{M}(\vec{r})=[n]$ and $\mathcal{P}(\vec{r})=[n]$. For $s \leqslant 0$, we give the necessary and sufficient conditions for $\mathcal{M}(\vec{r})=[n]-1$ and $\mathcal{P}(\vec{r})=[n]-1$.

Lemma 2.1 Let $\vec{r}=\left(r_{i}\right)_{i=1}^{n}$ be a sequence of real numbers with sum $s>0$. Let $j=m(\vec{r})$. For any $i=j+1, \ldots, n$, let $\vec{r}_{i}$ be the $i$-th cyclic permutation of $\vec{r}$. Then $m\left(\vec{r}_{i}\right)=n+j+1-i$.

Proof. It is easy to see $r_{i}+\ldots+r_{n}+r_{1}+\ldots+r_{k}<r_{i}+\ldots+r_{n}+r_{1}+\ldots r_{j}$ for any $k \in[j]-1$ and $r_{i}+\ldots+r_{n}+r_{1}+\ldots r_{k} \leqslant r_{i}+\ldots+r_{n}+r_{1}+\ldots r_{j}$ for any $k \in\{j, j+1, \ldots, i-1\}$. Assume 
that there is an index $k \in\{i, i+1, \ldots, n-1\}$ such that $r_{i}+\ldots+r_{k} \geqslant r_{i}+\ldots+r_{n}+r_{1}+\ldots+r_{j}$. Thus $r_{k+1}+\ldots+r_{n}+r_{1}+\ldots+r_{j} \leqslant 0 . j=m(\vec{r})$ implies $r_{1}+\ldots+r_{j} \geqslant r_{1}+\ldots+r_{k}$. So $0 \geqslant\left(r_{k+1}+\ldots+r_{n}\right)+r_{1}+\ldots+r_{j} \geqslant r_{1}+\ldots+r_{k}+\left(r_{k+1}+\ldots+r_{n}\right)=s>0$, a contradiction. We have $r_{i}+\ldots+r_{k}<r_{i}+\ldots+r_{n}+r_{1}+\ldots+r_{j}$ for any $k \in\{i, i+1, \ldots, n-1\}$. Hence $m\left(\vec{r}_{i}\right)=n+j+1-i$.

Theorem 2.2 Let $\vec{r}=\left(r_{i}\right)_{i=1}^{n}$ be a sequence of real numbers with sum $s>0$ and partial sums $\left(s_{i}\right)_{i=0}^{n}$. Let $j=m(\vec{r})$. Then $\mathcal{M}(\vec{r})=[n]$ if and only if $s_{j}-s_{i} \geqslant s$ for all $1 \leqslant i \leqslant j-1$.

Proof. For any $i \in[n]$, let $\vec{r}_{i}$ be the $i$-th cyclic permutation of $\vec{r}$. It is easy to see $m\left(\vec{r}_{i}\right) \neq 0$ since $s>0$. Lemma 2.1 tells us $m\left(\vec{r}_{i}\right)=n+j+1-i$ for any $i \in\{j+1, \ldots, n\}$.

Suppose $s_{j}-s_{i} \geqslant s$ for all $1 \leqslant i \leqslant j-1$. Consider the sequence $\vec{r}_{i}=\left(r_{i}, \ldots, r_{n}\right.$, $\left.r_{1}, \ldots, r_{i-1}\right)$ with $i \in[j]$. It is easy to see $r_{i}+\ldots+r_{k}<r_{i}+\ldots+r_{j}$ for any $k \in$ $\{i, i+1, \ldots, j-1\}$ and $r_{i}+\ldots+r_{k} \leqslant r_{i}+\ldots+r_{j}$ for any $k \in\{j, j+1, \ldots, n\}$. Assume that there is an index $k \in[i-1]$ such that $r_{i}+\ldots+r_{j}<r_{i}+\ldots+r_{n}+r_{1}+\ldots+r_{k}$. Thus $s_{j}-s_{k}=r_{k+1}+\ldots+r_{j}<s$, a contradiction. Hence $m\left(\vec{r}_{i}\right)=j+1-i$.

Conversely, suppose $\mathcal{M}(\vec{r})=[n]$. Let $A=\left\{i \mid s_{j}-s_{i}<s, 1 \leqslant i \leqslant j-1\right\}$. Assume $A \neq \emptyset$ and let $i=\min A$. Clearly $i+1 \leqslant j$. We consider the sequence $\vec{r}_{i+1}=$ $\left(r_{i+1}, \ldots, r_{n}, r_{1}, \ldots, r_{i}\right)$. Since $i \in A$, we have $r_{i+1}+\ldots+r_{j}<s=r_{i+1}+\ldots+r_{n}+r_{1}+\ldots+r_{i}$. It is easy to see $r_{i+1}+\ldots+r_{k}<r_{i+1}+\ldots+r_{j}$ for any $k \in\{i+1, i+2, \ldots, j-1\}$ and $r_{i+1}+\ldots+r_{k} \leqslant r_{i+1}+\ldots+r_{j}$ for any $k \in\{j, j+1, \ldots, n\}$. For every $k \in[i-1]$, we have $s_{j}-s_{k}=r_{k+1}+\ldots+r_{j} \geqslant s$ since $k \notin A$. So $r_{i+1}+\ldots+r_{j} \geqslant r_{i+1}+\ldots+r_{n}+r_{1}+\ldots r_{k}$. Hence $m\left(\vec{r}_{i+1}\right)=n=m\left(\vec{r}_{j+1}\right)$. So $\mathcal{M}(\vec{r}) \neq[n]$, a contradiction.

Lemma 2.3 Let $\vec{r}=\left(r_{i}\right)_{i=1}^{n}$ be a sequence of real numbers with sum $s \leqslant 0$. Let $j=m(\vec{r})$. Suppose $j \geqslant 1$. For any $i \in[j]$, let $\vec{r}_{i}$ be the $i$-th cyclic permutation of $\vec{r}$. Then $m\left(\vec{r}_{i}\right)=$ $j+1-i$.

Proof. It is easy to see $r_{i}+\ldots+r_{k}<r_{i}+\ldots+r_{j}$ for any $k \in\{i, i+1, \ldots, j-1\}$ and $r_{i}+\ldots+r_{k} \leqslant r_{i}+\ldots+r_{j}$ for any $k \in\{j, j+1, \ldots, n\}$. For any $k \in[i-1]$, we have $r_{k+1}+\ldots+r_{j}>0 \geqslant s$ since $j=m(\vec{r})$. This implies $0>r_{j+1}+\ldots+r_{n}+r_{1}+\ldots r_{k}$ and $r_{i}+\ldots+r_{j}>r_{i}+\ldots+r_{n}+r_{1}+\ldots r_{k}$. Note that $r_{i}+\ldots+r_{j}>0$ since $j=m(\vec{r})$. Hence $m\left(\vec{r}_{i}\right)=j+1-i$.

Theorem 2.4 Let $\vec{r}=\left(r_{i}\right)_{i=1}^{n}$ be a sequence of real numbers with sum $s \leqslant 0$ and partial sums $\left(s_{i}\right)_{i=0}^{n}$. Suppose $m(\vec{r})=j$. Then $\mathcal{M}(\vec{r})=[n]-1$ if and only if $s_{i}-s_{j}<s$ for all $j+1 \leqslant i \leqslant n-1$.

Proof. For any $i \in[n]$, let $\vec{r}_{i}$ be the $i$-th cyclic permutation of $\vec{r}$. It is easy to see $m\left(\vec{r}_{i}\right) \neq n$ since $s \leqslant 0$.

Suppose $s_{i}-s_{j}<s$ for all $j+1 \leqslant i \leqslant n-1$. Given an index $i \in\{j+1, j+2, \ldots, n\}$, we consider the sequence $\vec{r}_{i}=\left(r_{i}, \ldots, r_{n}, r_{1}, \ldots, r_{i-1}\right)$. It is easy to see $r_{i}+\ldots+r_{n}+r_{1}+$ $\ldots+r_{k}<r_{i}+\ldots+r_{n}+r_{1}+\ldots+r_{j}$ for any $k \in[j]-1$ and $r_{i}+\ldots+r_{n}+r_{1}+\ldots+r_{k} \leqslant$ $r_{i}+\ldots+r_{n}+r_{1}+\ldots+r_{j}$ for any $k \in\{j, j+1, \ldots, i-1\}$. For any $k \in\{i, i+1, \ldots, n-1\}$, 
since $s_{k}-s_{j}=r_{j+1}+\ldots+r_{k}<s$, we have $r_{k+1}+\ldots+r_{n}+r_{1}+\ldots+r_{j}>0$ and $r_{i}+\ldots+r_{k}<r_{i}+\ldots+r_{n}+r_{1}+\ldots+r_{j}$.

For $i \geqslant j+2$, note that $r_{i}+\ldots+r_{n}+r_{1}+\ldots+r_{j}>0$ since $j=m(\vec{r})$. Clearly, $r_{j+1}+\ldots+r_{n}+r_{1}+\ldots+r_{j}=s$. Hence $m\left(\vec{r}_{i}\right)=n+j+1-i$ for $i=j+2, \ldots, n$ and $m\left(\vec{r}_{j+1}\right)=0$. When $j \geqslant 1$, Lemma 2.3 tells us $m\left(\vec{r}_{i}\right)=j+1-i$ for any $i \in[j]$. Thus we have $\mathcal{M}(\vec{r})=[n]-1$.

Conversely, suppose $\mathcal{M}(\vec{r})=[n]-1$. Let $A=\left\{i \mid s_{i}-s_{j} \geqslant s, j+1 \leqslant i \leqslant n\right\}$. Note that $n \notin A$ if $j \geqslant 1$; otherwise $n \in A$. So, assume $A \backslash\{n\} \neq \emptyset$ and let $i=\max A \backslash\{n\}$. Clearly $j+1 \leqslant i \leqslant n-1$. We consider the sequence $\vec{r}_{i+1}=\left(r_{i+1}, \ldots, r_{n}, r_{1}, \ldots, r_{i}\right)$. It is easy to see $r_{i+1}+\ldots+r_{n}+r_{1}+\ldots+r_{k}<r_{i+1}+\ldots+r_{n}+r_{1}+\ldots+r_{j}$ for any $k \in[j]-1$ and $r_{i+1}+\ldots+r_{n}+r_{1}+\ldots+r_{k} \leqslant r_{i+1}+\ldots+r_{n}+r_{1}+\ldots+r_{j}$ for any $k \in\{j, j+1, \ldots, i\}$. For any $k \in\{i+1, i+2, \ldots, n-1\}$, we have $s_{k}-s_{j}=r_{j+1}+\ldots+r_{k}<s$ since $k \notin A$ and $r_{i+1}+\ldots+r_{k}<r_{i+1}+\ldots+r_{n}+r_{1}+\ldots+r_{j}$. Since $i \in A$, we have $r_{i+1}+\ldots+r_{n}+r_{1}+\ldots+r_{j} \leqslant 0$. Hence $m\left(\vec{r}_{i+1}\right)=0=m\left(\vec{r}_{j+1}\right)$ and $\mathcal{M}(\vec{r}) \neq[n]-1$, a contradiction.

For any sequence of real numbers $\vec{r}=\left(r_{i}\right)_{i=1}^{n}$ with partial sums $\left(s_{i}\right)_{i=1}^{n}$, we define a linear order $\prec_{\vec{r}}$ on the set $[n]$ by the following rules:

for any $i, j \in[n], i \prec_{\vec{r}} j$ if either $(1) s_{i}<s_{j}$ or $(2) s_{i}=s_{j}$ and $i>j$.

The sequence formed by writing elements in the set $[n]$ in the increasing order with respect to $\prec_{\vec{r}}$ is denoted by $\pi(\vec{r})=\left(\pi_{1}, \pi_{2}, \ldots, \pi_{n}\right)$. Note that $\pi(\vec{r})$ also can be viewed as a bijection from the set $[n]$ to itself.

Lemma 2.5 Let $\vec{r}=\left(r_{i}\right)_{i=1}^{n}$ be a sequence of real numbers with sum $s>0$. Let $\pi(\vec{r})$ be the linear order on the set $[n]$ with respect to $\prec_{\vec{r}}$. Given an index $j \in[n]$, let $\vec{r}_{j+1}=$ $\left(r_{j+1}, \ldots, r_{n}, r_{1}, \ldots, r_{j}\right)$. Then

(1) for any $j \prec_{\vec{r}} i$ we have $r_{j+1}+\ldots+r_{n}+r_{1}+\ldots+r_{i}>0$ if $i<j ; r_{j+1}+\ldots+r_{i}>0$ if $i>j$.

(2) Suppose $\pi(k)=j$ for some $k \in[n]$. We have $p\left(\vec{r}_{j+1}\right) \geqslant n-k+1$.

Proof. (1) $j \prec_{\vec{r}} i$ implies either (I) $s_{j}<s_{i}$ or (II) $s_{j}=s_{i}$ and $j>i$. Hence, we consider two cases as follows.

Case I. $s_{j}<s_{i}$. For $i>j$, it is easy to see $r_{j+1}+\ldots+r_{i}>0$. For $i<j$, we have $r_{i+1}+\ldots+r_{j}<0$. Hence $r_{j+1}+\ldots+r_{n}+r_{1}+\ldots r_{i}=s-r_{i+1}-\ldots-r_{j}>s>0$.

Case II. $s_{j}=s_{i}$ and $j>i$. We have $r_{i+1}+\ldots+r_{j}=0$ and $r_{j+1}+\ldots+r_{n}+r_{1}+\ldots+r_{i}=$ $s>0$.

(2) Note that $r_{j+1}+\ldots+r_{n}+r_{1} \ldots+r_{j}=s>0$. Hence $p\left(\vec{r}_{j+1}\right) \geqslant n-k+1$.

Lemma 2.6 Let $\vec{r}=\left(r_{i}\right)_{i=1}^{n}$ be a sequence of real numbers with sum $s>0$ and partial sums $\left(s_{i}\right)_{i=1}^{n}$. Let $\pi(\vec{r})$ be the linear order on the set $[n]$ with respect to $\prec_{\vec{r}}$. Let $j \in[n]$ and $\vec{r}_{j+1}$ be the $(j+1)$-th cyclic permutation of $\vec{r}$. Suppose $s_{j}-s_{i} \notin(0, s)$ for all $1 \leqslant i \leqslant j-1$ and $\pi(k)=j$ for some $k \in[n]$. Then $p\left(\vec{r}_{j+1}\right)=n-k+1$. 
Proof. For any $i \prec_{\vec{r}} j$, we discuss the following two case.

Case 1. $s_{i}<s_{j}$. For $i>j$, it is easy to see $r_{j+1}+\ldots+r_{i}<0$. For $i<j$, we have $s_{j}-s_{i}=r_{i+1}+\ldots+r_{j} \geqslant s$ since $s_{j}-s_{i}>0$ and $s_{j}-s_{i} \notin(0, s)$. Hence $r_{j+1}+\ldots+r_{n}+r_{1}+\ldots r_{i}=s-r_{i+1}-\ldots-r_{j} \leqslant 0$.

Case 2. $s_{i}=s_{j}$ and $i>j$. Clearly, we have $r_{j+1}+\ldots+r_{i}=0$.

By Lemma 2.5, we have $p\left(\vec{r}_{j+1}\right)=n+1-k$ since $\pi(k)=j$.

Theorem 2.7 Let $\vec{r}=\left(r_{i}\right)_{i=1}^{n}$ be a sequence of real numbers with sum $s>0$ and partial sums $\left(s_{i}\right)_{i=1}^{n}$. Then $\mathcal{P}(\vec{r})=[n]$ if and only if $s_{j}-s_{i} \notin(0, s)$ for any $1 \leqslant i<j \leqslant n$.

Proof. Let $\pi(\vec{r})$ be the linear order on the set $[n]$ with respect to $\prec_{\vec{r}}$. Suppose $s_{j}-s_{i} \notin$ $(0, s)$ for any $1 \leqslant i<j \leqslant n$. Lemma 2.6 implies $p\left(\vec{r}_{\pi(k)+1}\right)=n+1-k$ for all $k \in[n]$. Hence $\mathcal{P}(\vec{r})=[n]$.

Conversely, suppose $\mathcal{P}(\vec{r})=[n]$. Lemma 2.5 tells us $p\left(\vec{r}_{\pi(k)+1}\right) \geqslant n-k+1$ for all $k \in[n]$. Let $A_{k}=\left\{i \mid 0<s_{\pi(k)}-s_{i}<s, 1 \leqslant i<\pi(k)\right\}$ for any $k \in[n]$. Assume that $A_{k} \neq \emptyset$ for some $k \in[n]$. Let $\bar{k}=\min \left\{k \mid A_{k} \neq \emptyset\right\}$. By Lemma 2.6, we have $p\left(\vec{r}_{\pi(k)+1}\right)=n-k+1$ for any $k<\bar{k}$. Suppose $\pi(\bar{k})=j$. We consider the sequence $\vec{r}_{j+1}=\left(r_{j+1}, \ldots, r_{n}, r_{1}, \ldots, r_{j}\right)$. Let $i \in A_{\bar{k}}$. Since $s_{j}-s_{i}>0$, we have $s_{j}>s_{i}$. Thus $i \prec_{\vec{r}} j$ and $r_{j+1}+\ldots+r_{n}+r_{1}+\ldots+r_{i}=s-r_{i+1}-\ldots-r_{j}>0$ since $s_{j}-s_{i}<s$. By Lemma 2.5, we get $p\left(\vec{r}_{\pi(\bar{k})+1}\right) \geqslant n-\bar{k}+2$. Hence $n-\bar{k}+1 \notin \mathcal{P}(\vec{r})$, a contradiction.

Lemma 2.8 Let $\vec{r}=\left(r_{i}\right)_{i=1}^{n}$ be a sequence of real numbers with sum $s \leqslant 0$ and partial sums $\left(s_{i}\right)_{i=1}^{n}$. Let $\pi(\vec{r})$ be the linear order on the set $[n]$ with respect to $\prec_{\vec{r}}$. Given an index $j \in[n]$, let $\vec{r}_{j+1}=\left(r_{j+1}, \ldots, r_{n}, r_{1}, \ldots, r_{j}\right)$. Then

(1) for any $i \prec_{\vec{r}} j$, we have $r_{j+1}+\ldots+r_{n}+r_{1}+\ldots+r_{i} \leqslant 0$ if $i<j ; r_{j+1}+\ldots+r_{i} \leqslant 0$ if $i>j$.

(2) Suppose $\pi(k)=j$ for some $k \in[n]$. We have $p\left(\vec{r}_{j+1}\right) \leqslant n-k$.

Proof. (1) $i \prec_{\vec{r}} j$ implies either (I) $s_{i}<s_{j}$ or (II) $s_{i}=s_{j}$ and $i>j$. Hence, we consider two cases as follows.

Case I. $s_{i}<s_{j}$. For $i>j$, it is easy to see $r_{j+1}+\ldots+r_{i}<0$. For $i<j$, we have $r_{i+1}+\ldots+r_{j}>0$. Hence $r_{j+1}+\ldots+r_{n}+r_{1}+\ldots r_{i}=s-r_{i+1}-\ldots-r_{j}<0$.

Case II. $s_{i}=s_{j}$ and $i>j$. We have $r_{j+1}+\ldots+r_{i}=0$.

(2) Note that $r_{j+1}+\ldots+r_{n}+r_{1}+\ldots+r_{j}=s \leqslant 0$. Hence $p\left(\vec{r}_{j+1}\right) \leqslant n-k$.

Lemma 2.9 Let $\vec{r}=\left(r_{i}\right)_{i=1}^{n}$ be a sequence of real numbers with sum $s \leqslant 0$ and partial sums $\left(s_{i}\right)_{i=1}^{n}$. Let $\pi(\vec{r})$ be the linear order on the set $[n]$ with respect to $\prec_{\vec{r}}$. Let $j \in[n]$ and $\vec{r}_{j+1}$ be the $(j+1)$-th cyclic permutation of $\vec{r}$. Suppose $s_{j}-s_{i} \notin[s, 0]$ for all $1 \leqslant i \leqslant j-1$ and $\pi(k)=j$ for some $k \in[n]$. Then $p\left(\vec{r}_{j+1}\right)=n-k$.

Proof. Clearly, $r_{j+1}+\ldots+r_{n}+r_{1}+\ldots+r_{j}=s \leqslant 0$. For any $j \prec_{\vec{r}} i$, we claim $s_{i}>s_{j}$. Otherwise $s_{i}=s_{j}$, then $i<j$ and $s_{j}-s_{i}=0$, a contradiction.

For $i>j$, it is easy to see $r_{j+1}+\ldots+r_{i}>0$. For $i<j$, we have $s_{j}-s_{i}<s$ since $s_{j}-s_{i}<0$ and $s_{j}-s_{i} \notin[s, 0]$. So $r_{j+1}+\ldots+r_{n}+r_{1}+\ldots r_{i}=s-r_{i+1}-\ldots-r_{j}>0$. By Lemma 2.5, we have $p\left(\vec{r}_{j+1}\right)=n-k$. 
Theorem 2.10 Let $\vec{r}=\left(r_{i}\right)_{i=1}^{n}$ be a sequence of real numbers with sum $s \leqslant 0$ and partial sums $\left(s_{i}\right)_{i=1}^{n}$. Then $\mathcal{P}(\vec{r})=[n]-1$ if and only if $s_{j}-s_{i} \notin[s, 0]$ for all $1 \leqslant i<j \leqslant n$.

Proof. Let $\pi(\vec{r})$ be the linear order on the set $[n]$ with respect to $\prec_{\vec{r}}$. Suppose $s_{j}-s_{i} \notin$ $[s, 0]$ for all $1 \leqslant i<j \leqslant n$. Lemma 2.9 implies $p\left(\vec{r}_{\pi(k)+1}\right)=n-k$ for all $k \in[n]$. Hence $\mathcal{P}(\vec{r})=[n]-1$.

Conversely, suppose $\mathcal{P}(\vec{r})=[n]$. Lemma 2.8 tells us $p\left(\vec{r}_{\pi(k)+1}\right) \leqslant n-k$ for all $k \in[n]$. Let $A_{k}=\left\{i \mid s \leqslant s_{\pi(k)}-s_{i} \leqslant 0,1 \leqslant i \leqslant \pi(k)-1\right\}$ for any $k \in[n]$. Assume that $A_{k} \neq \emptyset$ for some $k \in[n]$. Let $\bar{k}=\max \left\{k \mid A_{k} \neq \emptyset\right\}$. By Lemma 2.9, we have $p\left(\vec{r}_{\pi(k)+1}\right)=n-k$ for any $k>\bar{k}$. Suppose $\pi(\bar{k})=j$. We consider the sequence $\vec{r}_{j+1}=\left(r_{j+1}, \ldots, r_{n}, r_{1}, \ldots, r_{j}\right)$. Let $i \in A_{\bar{k}}$. Since $s_{j}-s_{i} \leqslant 0$, we have $s_{j} \leqslant s_{i}$. Thus $j \prec_{\vec{r}} i$ and $r_{j+1}+\ldots+r_{n}+r_{1}+\ldots+r_{i}=$ $s-r_{i+1}-\ldots-r_{j} \leqslant 0$ since $s_{j}-s_{i} \geqslant s$. By Lemma 2.8, we get $p\left(\vec{r}_{j+1}\right) \leqslant n-\bar{k}-1$. Hence $n-\bar{k} \notin \mathcal{P}(\vec{r})$, a contradiction.

Now, we consider integer sequences. Taking $s=1$ in Theorems 2.2 and 2.7, we immediately obtain the following results.

Corollary 2.11 Let $\vec{r}=\left(r_{i}\right)_{i=1}^{n}$ be a sequence of integers with sum 1 . Then $\mathcal{M}(\vec{r})=$ $\mathcal{P}(\vec{r})=[n]$.

Taking $s=0$ in Theorems 2.4 and 2.10, we have the following corollary.

Corollary 2.12 Let $\vec{r}=\left(r_{i}\right)_{i=1}^{n}$ be a sequence of integers with sum 0 and the partial sums are all distinct. Then $\mathcal{M}(\vec{r})=\mathcal{P}(\vec{r})=[n]-1$.

Given a sequence $\vec{r}=\left(r_{1}, \ldots, r_{n}\right)$, recall that $\vec{r}_{\sigma}=\left(r_{\sigma(1)}, \ldots, r_{\sigma(n)}\right)$ for any $\sigma \in \mathfrak{S}_{n}$. For any $i \in[n+1]-1, N(\vec{r} ; i)$ ( resp. $\Pi(\vec{r} ; i))$ denotes the number of permutations $\sigma$ in $\mathfrak{S}_{n}$ such that $p\left(\vec{r}_{\sigma}\right)=i$ (resp. $m\left(\vec{r}_{\sigma}\right)=i$ ).

Corollary 2.13 Let $\vec{r}=\left(r_{i}\right)_{i=1}^{n}$ be a sequence of real numbers with sum $s$.

(1) Suppose $s>0$. Then $\Pi(\vec{r} ; i)=N(\vec{r} ; i)=(n-1)$ ! for all $i \in[n]$, if and only if $\sum_{k \in I} r_{k} \notin(0, s)$ for all $\emptyset \neq I \subseteq[n]$.

(2) Suppose $s \leqslant 0$. Then $\Pi(\vec{r} ; i)=N(\vec{r} ; i)=(n-1)$ ! for all $i \in[n]-1$ if and only if $\sum_{k \in I} r_{k} \notin[s, 0]$ for all $\emptyset \neq I \subset[n]$.

Proof. (1) Let $\sigma$ and $\tau$ be two permutations in $\mathfrak{S}_{n}$. We say $\sigma$ and $\tau$ are cyclicly equivalent, denoted by $\sigma \sim \tau$, if there is an index $i \in[n]$ such that $\tau=(\sigma(i), \ldots, \sigma(n), \sigma(1), \ldots, \sigma(i-$ $1))$. Hence, given a permutation $\sigma \in \mathfrak{S}_{n}$, we define a set $E Q(\sigma)$ as $E Q(\sigma)=\left\{\tau \in \mathfrak{S}_{n} \mid\right.$ $\tau \sim \sigma\}$. We say the set $E Q(\sigma)$ is an equivalence class of the set $\mathfrak{S}_{n}$. Clearly $|E Q(\sigma)|=n$ for any $\sigma \in \mathfrak{S}_{n}$.

Suppose $\sum_{k \in I} r_{k} \notin(0, s)$ for all $\emptyset \neq I \subseteq[n]$. For any $1 \leqslant i \leqslant n$, by Theorems 2.2( resp. Theorem 2.7), every equivalence class contains exactly one permutation $\sigma$ such that $m\left(\vec{r}_{\sigma}\right)=i\left(\right.$ resp. $\left.p\left(\vec{r}_{\sigma}\right)=i\right)$. Hence, $\Pi(\vec{r} ; i)=N(\vec{r} ; i)=\frac{n !}{n}=(n-1)$ !. 
Fix a permutation $\sigma \in \mathfrak{S}_{n}$. Let $\bar{s}_{0}=0, \bar{s}_{1}=r_{\sigma(1)}, \bar{s}_{2}=r_{\sigma(1)}+r_{\sigma(2)}, \ldots, \bar{s}_{n}=r_{\sigma(1)}+$ $r_{\sigma(2)}+\ldots+r_{\sigma(n)}$. Let $j$ to be the largest index $i$ with $\bar{s}_{i}=\min _{0 \leqslant k \leqslant n} \bar{s}_{k}$. Consider the permutation $\tau=(\sigma(j+1), \ldots, \sigma(n), \sigma(1), \ldots, \sigma(j))$. Then $\tau \in E Q(\sigma)$ and $p\left(\vec{r}_{\tau}\right)=n$. Thus there is at least one element $\tau \in E Q(\sigma)$ such that $p\left(\vec{r}_{\tau}\right)=n$ and $N(\vec{r} ; n) \geqslant(n-1)$ !. Let $j^{\prime}$ to be the smallest index $i$ with $\bar{s}_{i}=\max _{0 \leqslant k \leqslant n} \bar{s}_{k}$. Consider the permutation $\tau^{\prime}=$ $\left(\sigma\left(j^{\prime}+1\right), \ldots, \sigma(n), \sigma(1), \ldots, \sigma\left(j^{\prime}\right)\right)$. Then $\tau^{\prime} \in E Q(\sigma)$ and $m\left(\vec{r}_{\tau^{\prime}}\right)=n$. Thus there is at least one element $\tau^{\prime} \in E Q(\sigma)$ such that $m\left(\vec{r}_{\tau^{\prime}}\right)=n$ and $\Pi(\vec{r} ; n) \geqslant(n-1)$ !.

Suppose $\Pi(\vec{r} ; i)=N(\vec{r} ; i)=(n-1)$ ! for any $i \in[n]$. Particularly, $\Pi(\vec{r} ; n)=N(\vec{r} ; n)=$ $(n-1)$ !. Assume that there exists a proper subset $I$ of $[n]$ such that $0<\sum_{k \in I} r_{k}<s$. Let $A=\left\{k \in I \mid r_{k} \leqslant 0\right\}, a=|A|$ and $j=|I|$. Suppose $I=\left\{i_{1}, \ldots, i_{a}, i_{a+1} \ldots, i_{j}\right\}$, where $i_{k} \in A$ for every $k \in[1, a]$. Let $J=[n] \backslash I, B=\left\{k \in J \mid r_{k} \leqslant 0\right\}$ and $b=|B|$. Suppose $J=$ $\left\{i_{j+1}, \ldots, i_{j+b}, i_{j+b+1}, \ldots, i_{n}\right\}$, where $i_{j+k} \in B$ for every $k \in[1, b]$. Let $\sigma$ be a permutation in $\mathfrak{S}_{n}$ such that $\sigma(k)=i_{k}$ for any $k \in[n]$. Note that $0<\sum_{k=1}^{j} r_{\sigma(k)}=\sum_{k \in I} r_{k}<s$. Thus we have $m\left(\vec{r}_{\sigma}\right)=n$. Consider another permutation $\tau=(\sigma(j+1), \ldots, \sigma(n), \sigma(1), \ldots, \sigma(j))$. It is easy to see $\sigma \sim \tau$ and $m\left(\vec{r}_{\tau}\right)=n$. Hence $\Pi(\vec{r} ; n)>(n-1)$ !, a contradiction. Let $\sigma^{\prime}=(\sigma(n), \sigma(n-1), \ldots, \sigma(1))$ and $\tau^{\prime}=(\tau(n), \tau(n-1), \ldots, \tau(1))$. Then $\sigma^{\prime} \sim \tau^{\prime}$ and $p\left(\vec{r}_{\sigma^{\prime}}\right)=p\left(\vec{r}_{\tau^{\prime}}\right)=n$. Hence $N(\vec{r} ; n)>(n-1)$ !, a contradiction.

(2) Suppose $\sum_{k \in I} r_{k} \notin[s, 0]$ for all $\emptyset \neq I \subset[n]$. Similar to the proof of Corollary 2.13 (1), we can obtain the results as desired.

Fix a permutation $\sigma \in \mathfrak{S}_{n}$. Let $\bar{s}_{0}=0, \bar{s}_{1}=r_{\sigma(1)}, \bar{s}_{2}=r_{\sigma(1)}+r_{\sigma(2)}, \ldots, \bar{s}_{n}=r_{\sigma(1)}+$ $r_{\sigma(2)}+\ldots+r_{\sigma(n)}$. Let $j$ to be the largest index $i$ with $\bar{s}_{i}=\max _{0 \leqslant k \leqslant n} \bar{s}_{k}$. Consider the permutation $\tau=(\sigma(j+1), \ldots, \sigma(n), \sigma(1), \ldots, \sigma(j))$. Clearly, $\tau \in E Q(\sigma)$ and $m\left(\vec{r}_{\tau}\right)=$ $p\left(\vec{r}_{\tau}\right)=0$. So there is at least one element $\tau \in E Q(\sigma)$ such that $m\left(\vec{r}_{\tau}\right)=p\left(\vec{r}_{\tau}\right)=0$. Thus $N(\vec{r} ; 0) \geqslant(n-1)$ ! and $\Pi(\vec{r} ; 0) \geqslant(n-1)$ !.

Suppose $\Pi(\vec{r} ; i)=N(\vec{r} ; i)=(n-1)$ ! for any $i \in[n]-1$. Particularly, $\Pi(\vec{r} ; 0)=$ $N(\vec{r} ; 0)=(n-1)$ !. Assume that there exists a proper subset $I$ of $[n]$ such that $s \leqslant \sum_{k \in I} r_{k} \leqslant$ 0 . Let $A=\left\{k \in I \mid r_{k} \leqslant 0\right\}, a=|A|$ and $j=|I|$. Suppose $I=\left\{i_{1}, \ldots, i_{a}, i_{a+1} \ldots, i_{j}\right\}$, where $i_{k} \in A$ for every $k \in[1, a]$. Let $J=[n] \backslash I, B=\left\{k \in J \mid r_{k} \leqslant 0\right\}$ and $b=|B|$. Suppose $J=\left\{i_{j+1}, \ldots, i_{j+b}, i_{j+b+1}, \ldots, i_{n}\right\}$, where $i_{j+k} \in B$ for every $k \in$ $[1, b]$. Let $\sigma$ be a permutation in $\mathfrak{S}_{n}$ such that $\sigma(k)=i_{k}$ for any $k \in[n]$. Note that $\sum_{k=1}^{j} r_{\sigma(k)}=\sum_{k \in I} r_{k} \leqslant 0$. Thus we have $m\left(\vec{r}_{\sigma}\right)=0$. Consider another permutation $\tau=$ $(\sigma(j+1), \ldots, \sigma(n), \sigma(1), \ldots, \sigma(j))$. Then $\sum_{k=1}^{n-j} r_{\tau(k)}=s-\sum_{k \in I} r_{k} \leqslant 0$ since $\sum_{k \in I} r_{k} \geqslant s$. So $m\left(\vec{r}_{\tau}\right)=0$. Note that $\sigma \sim \tau$. Hence $\Pi(\vec{r} ; 0)>(n-1)$ !, a contradiction. It is easy to see $p\left(\vec{r}_{\sigma}\right)=p\left(\vec{r}_{\tau}\right)=0$. Hence $N(\vec{r} ; 0)>(n-1)$ !, a contradiction. 


\section{$3 \quad$ More general cases}

In this section, we consider more general cases and study furthermore generalizations for properties of cyclic permutations of a sequence $\vec{r}=\left(r_{i}\right)_{i=1}^{n}$.

Theorem 3.1 Let $\theta$ be a real number and $\vec{r}=\left(r_{i}\right)_{i=1}^{n}$ a sequence of real numbers with sum $s>n \theta$ and partial sums $\left(s_{i}\right)_{i=0}^{n}$. Then

(1) $\mathcal{M}(\vec{r} ; \theta)=[n]$ if and only if $s_{j}-s_{i} \geqslant s-(n-j+i) \theta$ for all $1 \leqslant i \leqslant j-1$, where $j=m(\vec{r} ; \theta)$;

(2) $\mathcal{P}(\vec{r} ; \theta)=[n]$ if and only if $s_{j}-s_{i} \notin((j-i) \theta, s-(n+i-j) \theta)$ for all $1 \leqslant i<j \leqslant n$, where the notation $((j-i) \theta, s-(n+i-j) \theta)$ denote the set of all real numbers $x$ satisfying $(j-i) \theta<x<s-(n+i-j) \theta$.

Proof. (1) Consider the sequence $\vec{v}=\left(r_{1}-\theta, \ldots, r_{n}-\theta\right)$. It is easy to see that (I) $\sum_{i=1}^{n} \vec{v}_{i}=$ $s-n \theta>0 ;(\mathrm{II}) j=m(\vec{r} ; \theta)$ if and only if $j=m(\vec{v}) ;(\mathrm{III})\left(s_{j}-j \theta\right)-\left(s_{i}-i \theta\right) \geqslant s-n \theta>0$ for all $1 \leqslant i \leqslant j-1$. By Theorem 2.2 , we obtain the results as desired.

(2) Similar to the proof of Theorem 3.1(1), we can obtain the results in Theorem $3.1(2)$.

Similarly, considering $s \leqslant n \theta$, we can obtain the following results.

Theorem 3.2 Let $\theta$ be a real number and $\vec{r}=\left(r_{i}\right)_{i=1}^{n}$ a sequence of real numbers with sum $s \leqslant n \theta$ and partial sums $\left(s_{i}\right)_{i=0}^{n}$. Then

(1) $\mathcal{M}(\vec{r} ; \theta)=[n]-1$ if and only if $s_{i}-s_{j}<s-(n+j-i) \theta$ for all $j+1 \leqslant i \leqslant n-1$, where $j=m(\vec{r} ; \theta)$;

(2) $\mathcal{P}(\vec{r} ; \theta)=[n]-1$ if and only if $s_{j}-s_{i} \notin[s-(n+i-j) \theta,(j-i) \theta]$ for any $1 \leqslant i<j \leqslant n$, where the notation $[s-(n+i-j) \theta,(j-i) \theta]$ denote the set of all real numbers $x$ satisfying $s-(n+i-j) \theta \leqslant x \leqslant(j-i) \theta$.

\section{Acknowledgements}

The authors are thankful to the referees for their helpful comments to improve the paper.

\section{References}

[1] R. Altschul, Another proof for a combinatorial lemma in fluctuation theory, Math. Scand. 31 (1972), 123-126.

[2] E.S. Andersen, On sums of symmetrically dependent random variables, Skand. Aktuarietidskr. (1953) 123-138.

[3] G. Baxter, Notes for a seminar in stochastic processes, 1957. 
[4] A. Brandt, A generalization of a combinatorial theorem of Sparre Andersen about sums of random variables, Math. Scand. 9 (1961), 352-358.

[5] D. Callan, Pair them up! A visual approach to the Chung-Feller theorem, Coll. Math. J. 26(1995)196-198.

[6] D. Callan, Why are these equal? http://www.stat.wisc.edu/ callan/notes/

[7] K.L. Chung, W. Feller, On fluctuations in-coin tossing, Proc. Natl. Acad. Sci. USA 35 (1949) 605-608.

[8] S.P. Eu, T.S. Fu, Y.N. Yeh, Refined Chung-Feller theorems for lattice paths, J. Combin. Theory Ser. A 112 (2005) 143-162.

[9] S.P. Eu, S.C. Liu, Y.N. Yeh, Taylor expansions for Catalan and Motzkin numbers, Adv. Appl. Math. 29 (2002) 345-357.

[10] W. Feller, On combinatorial methods in fluctuation theory, The Harald Cramer Volume, Ed. Ulf Grenander, New York, 1959, 75-91. 143-162.

[11] R.L. Graham, D.E. Knuth, Oren Patashnik, Concrete Mathematics(2nd edition), Addison-Wesley, 1994.

[12] Ch. Hobby, R. Pyke, Remarks on the equivalence principle in fluctuation theory, Math. Scand. 12 (1963), 19-24.

[13] A. Huq, Generalized Chung-Feller Theorems for Lattice Paths (Thesis), http://arxiv.org/abs/0907.3254

[14] R.I. Jewett, K. A. Ross, Random walk on $\mathbb{Z}$, Coll. Math. J. 26(1995)196-198.

[15] S.C. Liu, Y. Wang, Y.N. Yeh, Chung-Feller property in view of generating functions, submitted

[16] J. Ma, Y.N. Yeh, Generalizations of The Chung-Feller Theorem I, Bull. Inst. Math. Acad. Sin. (N. S.) 4 (2009) 299-332.

[17] J. Ma, Y.N. Yeh, Generalizations of The Chung-Feller Theorem II, submitted.

[18] S.G. Mohanty, Lattice path counting and applications, New York: Academic Press, 1979.

[19] T.V. Narayana, Cyclic permutation of lattice paths and the Chung-Feller theorem, Skand. Aktuarietidskr. (1967) 23-30.

[20] T.V. Narayana, Lattice path combinatorics, with statistical applications, Toronto ; Buffalo : University of Toronto Press, c1979. 
[21] G.N. Raney, Functional composition patterns and power series reversion, Trans. Amer. Math. Soc., 94(1960) 441-451.

[22] L. Shapiro, Some open questions about random walks, involutions, limiting distributions, and generating functions, Adv. in Appl. Math. 27 (2001) 585-596.

[23] F. Spitzer, A combinatorial lemma and its application to probability theory, Trans. Amer. Math. Soc., 82(1956) 323-339.

[24] W.J. Woan, Uniform partitions of lattice paths and Chung-Feller Generalizations, Amer. Math. Monthly 108 (2001) 556-559. 